
SEQUENTTAL COMBINATION OF METHOTREXATE AND $1-\beta-D-$
155 ARABINOFURANOSYLCYTOSINE SHOWS SYNERGISTIC EFFCT ON THE GENERATION OF DNA STRAND BREAKS IN A HUMAN PROMYELOCYTIC LEUKEMIA CELL LINE

Akihiko Tanizawa, Masaru Kubota, Tetsuya Takimoto, Toshiyuki Kito, Yuichi Akiyama, Yukio Kiriyama and Haruki Mikawa Kyoto University, Department of Pediatrics, Kyoto, Japan.

It has recently been stated that MTX can cause DNA strand breaks. Ara-C, on the other hand, inhibits DNA repair synthesis after UV irradiation or exposure to chemical agents. Therefore, we investigated the accumulation of DNA strand breaks in a human promyelocytic leukemia cell line, HL-60, treated with methotrexate (MTX) and $1-\beta-D$-arabinofuranosylcytosine (Ara-C). The sequential treatment with MTX $(0.01-10 \mu \mathrm{M})$ then $\operatorname{Ara}-\mathrm{C}(10 \mu \mathrm{M})$ had a synergistic effect on the formation of DNA strand breaks, which was dependent on MTX concentration. On the other hand, when Ara-C preceded MTX, no such synergism was observed. The addition of both preceded $\mathrm{MTX}$, no such synergism was observed. The addition of both
thymidine $(10 \mu \mathrm{M})$ and hypoxanthine $(100 \mu \mathrm{M})$ to this system, but not thymidine or hypoxanthine alone, abolished the synergism. Pretreatment with MTX augmented the generation of $1-\beta-D$-arabinofuranosylcytosine 5'-triphosphate and the incorporation of Ara-C into DNA. Hluwever, these augmentation did not necessarily correlate with the amount of strand breaks. Whatever the underlying mechanism of this synergism is, our present data provide one possible biochemical basis for sequential MTX and Ara$C$ therapy.

ANALYSIS OF MOLECULAR STRUCTURE OF RAT

156 PHOSPHORTBOSYLPYROPHOSPHATE SYNTHETASE GENES.

Masamiti Tatibana, Masanori Taira, Sumio Ishijima,

Kazuko Kita, Hirieaki Shimada, Kazumi Yamada and

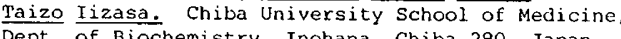

Dept. of Biochemistry, Inohana, Chiba 280, Japan.

Phosphoribosylpyrophosphate (PRPP) synthetase catalyzes the reaction: Rib-5-P+ATP $\Rightarrow$ PRPP+AMP. The mammalian enzyme exists as a complex aggregate and a $34 \mathrm{kDa}$ component has been identified as the catalytic subunit. A mutant enzyme in man with hyperactivity leads to clevated purine biosynthesis and eventually to gout. For further study of this enzyme, gene analysis is essential. We screened a cDNA library from rat Yoshida ascites sarcoma cells, using oligonucleotide probes based on the partial amino acid sequences of PRPP synthetase from rat liver. Two distinct clones were obtained and nucleotide sequencing revealed that both clones encode 317 amino acids. The deduced amino acid sequences of the two differ only by 13 residues, whereas the nucleotide sequences are relatively divergent ( 81 s identity in the coding regions). These results and amino acid sequencing data suggest the presence of two distinct isoforms of the JBC $202: 14067,1987$ ) JBC $262: 14867,1987)$. . To analyze the genomic gene structure, a rat genomic EMBL3 library was screened using PRS I and II CDNA probes and 16 clones were obtained, 15 of which were related to PRS I sequence and the other to PRS II. Studies of 7 overlapping PRS I-related clones showed the PRS I structural gene to span about $20 \mathrm{~kb}$. Four other PRS-related clones were quite distinct from the above 7 , regarding restriction maps. Thus the presence of additional gene or pseudogene loci for this enzyme is

suggested, in analogy to the four loci in humans (HGM 9:739,1987).

\section{ESTIMATION OF THE IN VITRO AND IN VIVO INHIBITORY} 157 EFFECTS OF ANTIFOLATES UPON THYMIDYLATE Jackman, Kathy Balmanno, A Hillary Calvert. Inst. Cancer Res. Drug Development Section, Sutton, Surrey, UK.

The ability of an antifolate to generate polyglutamates [via folyl polyglutamate sythetase (FPGS)] is an important determinant of its activity as this results in retention of the antimetabolite within the cells and may increase its affinity for the target enzyme. The estimation of TS activity in intact cells can be used to evaluate the retention of 5,8 dideazafolic acid (DAF) analogues by monitoring $\left[{ }^{3} \mathrm{H}\right] 2^{\prime}$-deoxyuridine metabolism in cells following the attainment of a state of dynamic equilibrium. CB3717 (2-amino 10-propargyl DAF) is an inhibitor of TS which has demonstrable clinical efficacy but also exhibits unpredictable toxicities. An in vitro structure activity study, with variations at positions 2 and 10 , gave results which ranged from weil retained (e.8. 2methyl, 10-H) through intermediate (e.g. CB3717) to essentially unretained methyl, 10-H) through intermediate (e.g. CB3717) to essentially unretained
(e.g. 2-methoxy, 10-propargyl). Retention within cells was found to (e.g. 2-methoxy, 10-propargyl). Retention within cells was found to
correlate with FPGS substrate activity but independent of both isolated correlate with FPGS substrate activity but independent of both isolated
TS inhibition and in vitro cytotoxicity. In mice it is possible to estimate the effect of analogues upon TS in ascitic tumour cells which are totally resistant to CB3717 due to the high circulating thymidine. CB3717 exhibited modest inhibition of TS in vive with only $43 \%$ inhibition $2 \mathrm{hrs}$ after the i.v. administration of the maximum tolerated dose $(200 \mathrm{mgs} / \mathrm{kg})$. Modification at the 2 position had a dramatic effect such that the dose required to at the dose $50 \%$ f desamino (2-H), and desamino (2-H), and 2 -methyl analogues respectively. Measuring the
drug/target interaction in intact cells has allowed us to identify analogues which are considerably more potent in vive TS inhibitors.

\section{THE MOMSV ENHANCER ON EXPRESSION OF THE APRT GENE.}

Milton W. Taylor, De Chu Tang, and Joo Park. Indiana Universitey, Department of Blology, Bloomington, IN 47405, USA.

The transcriptional unit of the CHO APRT gene was analyzed by deletional mutants at the $3^{\prime}$ and $5^{\prime}$ end of the gene, and by deletions in the intronic regions. Two sequences 5 , to the transcription start site are involved in gene regulation. One of these is a putative Spl binding region. The other region, within 52 bp of the transcription start site, as measured by DNA footprinting experiments contains a second protein binding site of 22 bp conserved in both mouse and $\mathrm{CHO}$ aprt genes, suggesting that it could be functionally important. This region is being analyzed by in vitro mutagenesis. The aprt structural gene and analyzed by $\frac{1 \mathrm{n}}{\mathrm{vitro}}$ mutagenesis. The aprt structural gene and
promoter was fused to the Moloney Murine Sarcoma Virus (MoMSV) promoter was fused to the Moloney Murine Sarcoma Virus (MoMSV)
LTR. These sequences contain the viral promoter and enhancer LTR. These sequences contain the viral promoter and enhancer
signals. The $5^{\prime}$ non-coding region of the aprt gene supresses signals. The $S^{\prime}$ non-coding reglion of the aprt gene supresses
activity of the Mo-MSV LTR. Deletion of the native aprt promoter allows Mo-MSV enhancer activity when the cells are grown in sodium butyrate. Under such conditions aprt enzyme activity 1 s enhanced 20-30 fold in both transient expression assays, and in stable transformants. The overproduction of APRT has no obvious detrimental effect on cell growth. \section{PNOSPRORIBOSYL, TRANSFERASE GENES. H. Hershey, A.
Sahota. and M.W. Taylor, IndIana University, Department of Blology, Bloomington, IN 47405} The mRNA of the APRT gene appears to be constitutively expressed and, thus, enzyme levels will be directly affected by mutations which alter translational efficiency. Accordingly, we have generated mutants altering the ATG start codon by in v1tro mutagenesis of the CHO apt gene (the $2.8 \mathrm{~kb}$ bam HI-XbaI fragment) on an M13 vector. Mutagenes1s was performed by the use of dut-ung- E. coll strains as described by Kunke1, et. al. (Methods dut- ${ }^{-}$ung E. coll strains as described by Kunkel, et. al. in Enzymology, 1987). Mutant genes are transferred to an
Epstein-Barr virus derived plasmid (p220.2). These plasmids grow Epstein-Barr virus derived plasmid (p220.2). These plas
episomally in human cell 1ines. We have also generated APRT- mutants in the HL-60 myelocytic leukemic cell line, which will be used as recipients for the plasmid. In addition to increasing our knowledge of translational effictency of non-cannonical start codons in eucaryotes, such a system can be used as a mutagen test system for potential mutagens and carcinogens, and also to analyze functional regions of the APRT protein.

\section{0 (ECTO-5'-NT) POSITIVE AND NEGATTVE \\ Linda F. Thompson and Julie M. Ruedi. Scripps Clinic and Research Foundation, Dept. of Immunology, La Jo1la, CA USA. \\ Human $T$ and $B$ lymphocytes, separated into ecto-5'-} NT positive and negative populations with goat anti-5'-NT antibodies and the fluorescence-activated cell sorter, were characterized in functional assays. Ecto-5 $-\mathrm{NT}^{-} \mathrm{T}$ cells proliferated as well as, or better than, ecto- $5^{\prime}-\mathrm{NT}^{+} \mathrm{T}$ cells af ter stimulation with phytohemagglutinin or in a mixed lymphocyte reaction. Ecto$5^{\prime}-\mathrm{NT}^{-} \mathrm{T}$ cells a1so provided more help for pokeweed mitogen (PWM)stimulated immunoglobulin synthesis than ecto-5 $5^{\prime}-\mathrm{NT}^{+t^{+}} \mathrm{T}$ cells. Therefore, the inability of ecto-5'-NT deficient lymphocytes from patients with immunodeficiency diseases to respond in these assays cannot be attributed solely to their reduced ecto-5'-NT

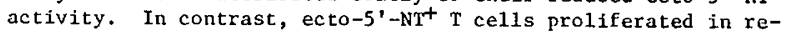
sponse to ten-fold lower doses of phorbol esters than ecto- $5^{\prime}-\mathrm{NT}^{-}$ $\mathrm{T}$ cells, suggesting that ecto- $5^{-}-\mathrm{NT}^{+} \mathrm{T}$ cells may utilize unique biochemical pathways of cellular activation or may have a prefer-

ential ability to respond to certain stimuli.
Ecto-5'-NT positive and negative B cells were tested for the ability to synthesize Igif and IgG af ter stimulation with PWM and Epstein Barr virus. Although both groups of cells synthesized

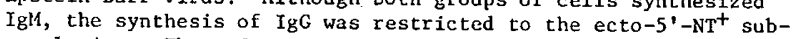
population. These data provide the first direct evidence that ecto-5'-NT is a marker for the functional maturation of human $B$ cells and demonstrate that ecto-5'-NT is different from other human $B$ cell surface antigens such as IgD, Leu 8 , and $H B-4$ which 